UCRL-JC-130921

PREPRINT

\title{
Phase Retrieval Techniques for Adaptive Optics
}

\author{
C.J. Carrano \\ S.S. Olivier \\ J.M. Brase \\ B.A. Macintosh \\ J.R. An
}

This paper was prepared for submittal to the SPIE 1998 Symposium on Astronomical Telescopes and Instrumentation

Kona, $\mathrm{HI}$

March 20-28, 1998

March 1998

This is a preprint of a paper intended for publication in a journal or proceedings. Since changes may be made before publication, this preprint is made available with the understanding that it will not be cited or reproduced without the permission of the author. 


\section{DISCLAIMER}

This document was prepared as an account of work sponsored by an agency of the Cinited States Government. Neither the United States Government nor the University of California nor any of their employees, makes any warranty, express or implied, or assumes any legal liability or responsibility for the accuracy, completeness, or usefulness of any information, apparatus, product, or process disclosed, or represents that its use w ould not infringe privately owned rights. Reference herein to any specific commercial products, process, or service by trade name, trademark, manufacturer, or otherwise, does not necessarily constitute or imply its endorsement, recommendation, or favoring by the United States Government or the University of California. The views and opinions of authors expressed herein do not necessarily state or reflect those of the United States Government or the University of California, and shall not be used for advertising or product endorsement purposes. 


\title{
Phase retrieval techniques for adaptive optics
}

\author{
C. J. Carrano ${ }^{a}$, S. S. Olivier ${ }^{a}$, J. M. Brase ${ }^{a}$, B. A. Macintosh ${ }^{a}$ and J. R. An ${ }^{a}$ \\ ${ }^{a}$ Lawrence Livermore National Laboratory, P.O. Box 808, Livermore, CA 94550
}

\begin{abstract}
We have developed and tested a method for minimizing static aberrations in adaptive optics systems. In order to correct the static phase aberrations, we need to measure the aberrations through the entire system. We have cmployed various phase retrieval algorithms to detect thesc aberrations. We have performed simulations of our experimental setup demonstrating that phase retrieval can improve the static aberrations to below the $20 \mathrm{~nm} \mathrm{rms}$ level, with the limiting factor being local turbulence in the AO system. Expcrimentally thus far, we have improved the static aberrations down to the $50 \mathrm{~nm}$ level, with the limiting factor being the ability to adjust the deformable mirror. This should be improved with better control algorithms now being implemented.
\end{abstract}

Keywords: adaptive optics, phase retrieval, phase diversity, optical system calibration

\section{INTRODUCTION}

The task of minimizing the static optical aberrations delivered to the science camera focal plane in an adaptive optics (AO) system is fundamental to the ability of the system to provide astronomical data of the highest possible quality. Furthermore, maintaining the accuracy of calibration during observations is of paramount importance in providing a slable PSF to the science camera, thereby allowing accurate discrimination between instrumental artifacts and real astronomical structure. Phase-diverse phase recovery techniques have been successfully applied to the general area of optical system calibration, including diagnosis of the aberrations of the Hubble Space Telescope. ${ }^{1}$ Application of these techniques to measurement of static phase errors for an adaptive optics system has also been recently investigated at the Starfire Optical Range. ${ }^{2}$

In this paper, we will discuss the use the phase retrieval techniques to obtain and maintain high-accuracy calibration of an adaptive optics system that uses both natural and sodium-layer laser guide stars. Results from simulations will be presented, along with results from experiments performed with the Lick Observatory adaptive optics system. ${ }^{3}$

\section{GENERAL METHOD FOR STATIC ABERRATION MINIMIZATION}

A block diagram of the Lick Observatory AO system is shown in Figure 1 on the next page. We have developed a general method for minimizing the static optical aberrations seen by the science camera detector consisting of the following steps:

- Insert a point source at the telescope focal plane.

- Adjust the deformable mirror (and relay optics) to optimize the science camera image.

- Measure the wavefront with the wavefront sensor (WFS) and use this as the system reference wavefront.

Other author information: (Send correspondence to C.J.C. or S.S.O)

C.J.C.: E-mail: carrano2@linl.gov

S.S.O.: E-mail: olivier1@llnl.gov 


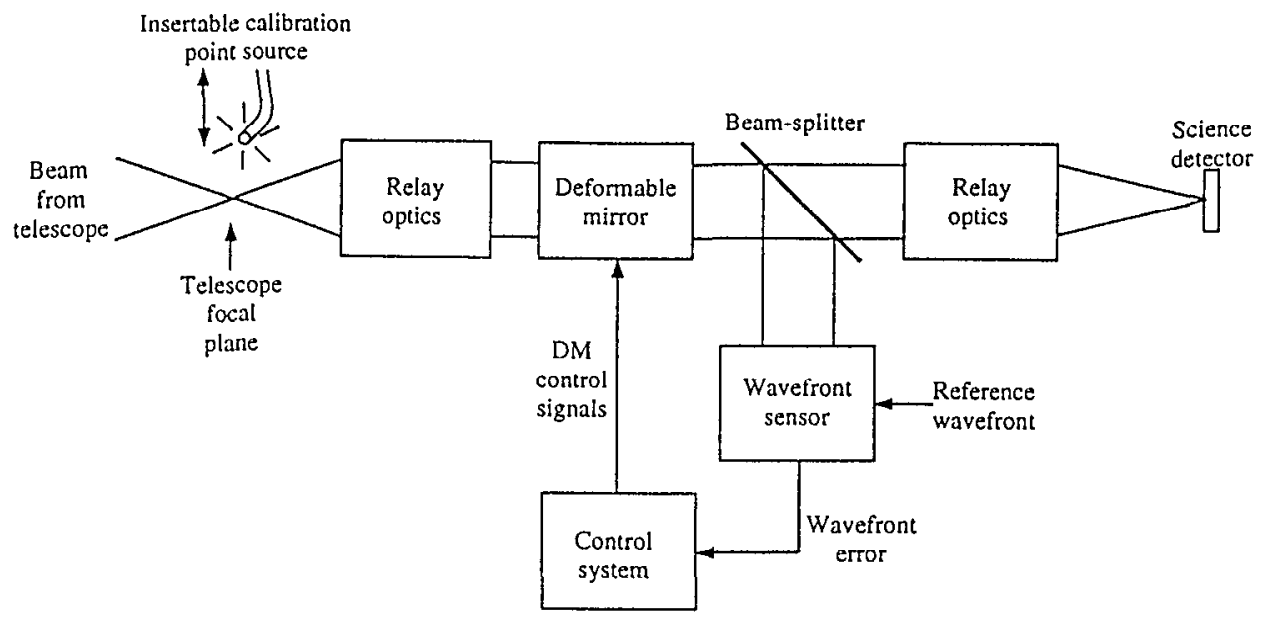

Figure 1. Block diagram of AO system.

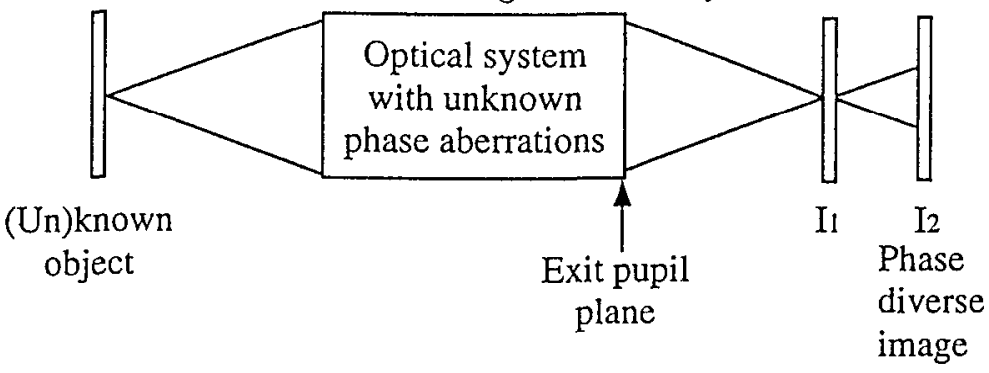

Figure 2. Block diagram of phase diversity concept.

\subsection{Optimization of the science camera image}

The general technique of phase retrieval can be used to determine the appropriate adjustments to the deformable mirror (DM). Using the science camera image, phase retrieval estimates the wavefront phase as seen through the entire system. The deformable mirror can then be adjusted to cancel the phase error measured by phase retrieval, thus improving the science camera image.

Another technique of obtaining the wavefront phase would be to measure it interferometrically. The advantage of phase retrieval over interferometric techniques is that phase retrieval requires no additional optical instrumentation or other system modification, with exception of moving the science camera to an out of focus position. It is often impractical to insert an interferometer at the science camera detector itself, especially for an IR camera where the detector is inside a dewar.

\section{PHASE RETRIEVAL AND PHASE-DIVERSE PHASE RETRIEVAL}

Phase retrieval techniques use a single intensity image, usually measured at the focal plane, and some knowledge of the pupil to estimate the aberrated wavefront. Phase retrieval techniques that use two or more images differing by a known wavefront phase (typically pure focus) are generally called phase-diverse phase retrieval if the object is known or phase diversity if the object is not known. This conccpt was originally proposed by Gonsalves. ${ }^{5}$ A block diagram of the concept is shown in Figure 2. The use of phase diversity algorithms allows both the aberrated wavefront and the object to be cstimated. For adaptive optics calibration, when the object is a point source, the algorithms can be simplified.

\subsection{Methods of (phase diverse) phase retrieval}

There are a number of algorithms and variations on algorithms that perform (phase-diverse) phase retrieval. We list some of them here along with their basic capabilities: 


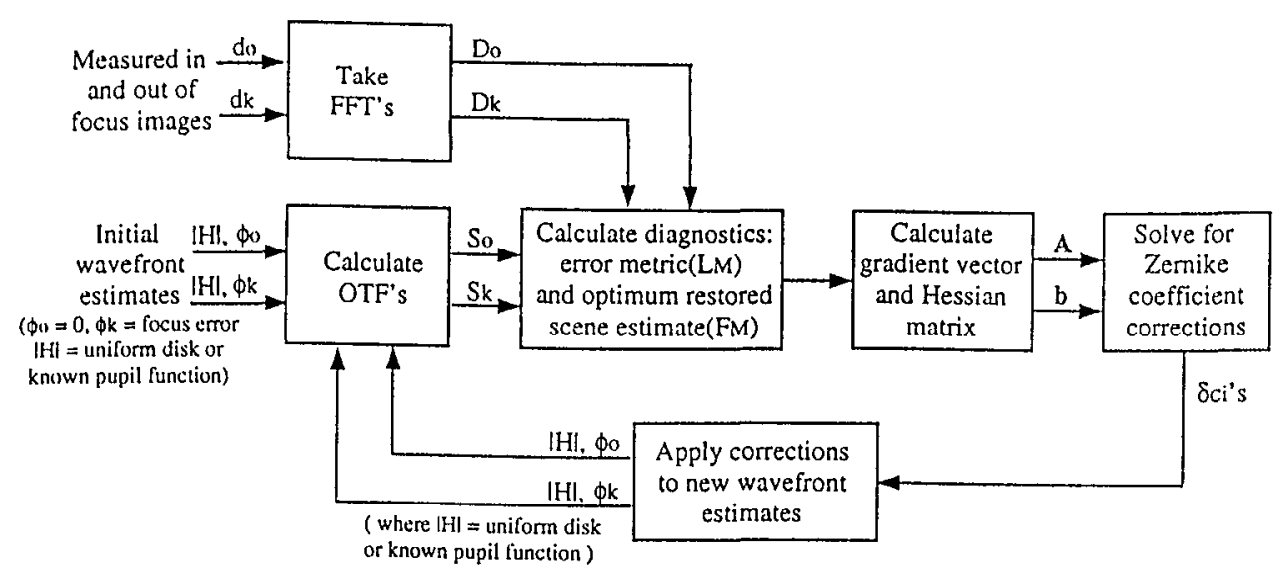

Figure 3. Block diagram of Least Squares method.

- Least squares methods; use a Gaussian noise $\operatorname{model}^{8}$; estimate wavefront directly and estimate object indirectly.

- Gradient search methods; can use a Gaussian or Poisson noise model $^{7}$; can assume object and estimate wavefront or jointly estimate object and wavefront.

- Iterative propagation or error-reduction methods ${ }^{4,6}$; estimate wavefront only; no object information estimated but usually imply a point source object.

In implementing the first two of these three methods, it is necessary to choose how to describe the wavefront. That is, what set of basis functions will best characterize the wavefront errors of interest. Example basis sets include Zernike polynomials, individual DM actuators, orthogonal DM modes, mirror segment piston, tips, and tilts, and individual phase points. The third method listed above typically estimates a map of individual phase points, which can be fitted by other basis sets if desired.

In the next three subscctions we givc a block diagram and a brief explanation of each method listed above. For more detailed explanations, see the references cited above.

\subsubsection{Least squares method}

A block diagram of this method is shown in Figure 3. In this method, we seek to minimize an error metric $\left(L_{M}\right)$ by finding the proper values for the basis set coefficients that we chose to describe the phase aberrations. This is done in an iterative fashion, where corrcctions to the coefficients $\left(\delta c_{i}\right.$ 's) are calculated such that the minimum of $L_{M}$ is found in the next iteration. The calculation involves solving a matrix equation of the form $A \cdot \delta c=b$, where $A$ is the Ilessian or second derivative matrix and $b$ is the gradient vector of $L_{M}$ with respect to the aberration parameters.

Run times for this method tend to slow down considerably as the basis set size increases or the image sizes increases.

\subsubsection{Gradient search methods with object known}

A block diagram of this method is shown in Figure 4. This algorithm begins by making an initial wavefront estimate (usually zero or flat) in terms of the chosen aberrations basis set. This set of aberration coefficients is fed to the conjugate gradient routine that will do line searches and result in an estimate of the actual aberration parameters. For the conjugate gradient routine to progress, it repeatedly calculates the log-likelihood function $\left(L_{M}\right)$ for the appropriate noise model, along with its gradient with respect to each aberration basis set coefficient. When $L_{M}$ has reached an acceptable level, the routine is terminated.

As the basis set size increases or the image size increases, this method tends to runs somewhat faster than the Least Squares methods. 


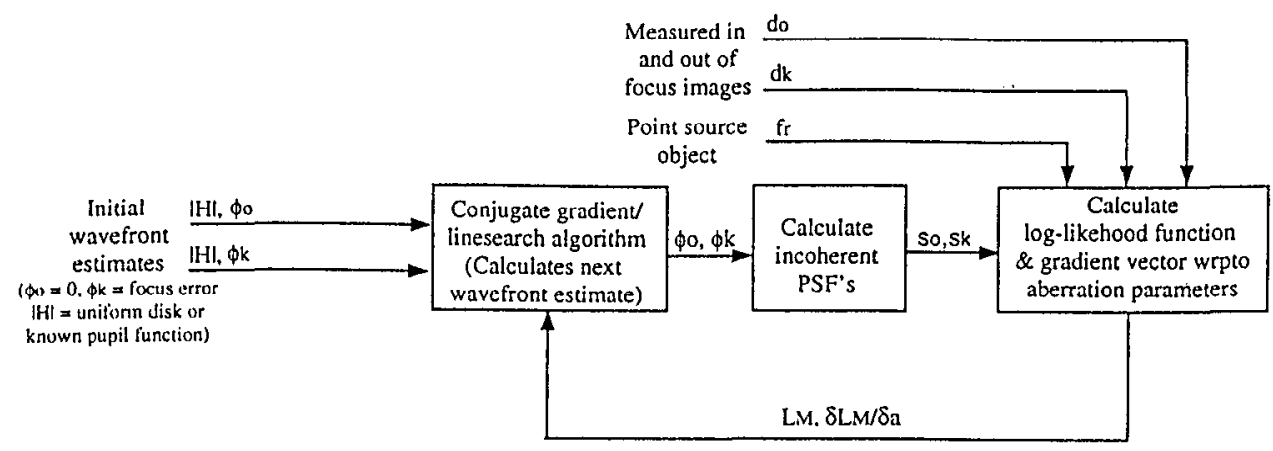

Figure 4. Block diagram of the Gradient Search method with known object.

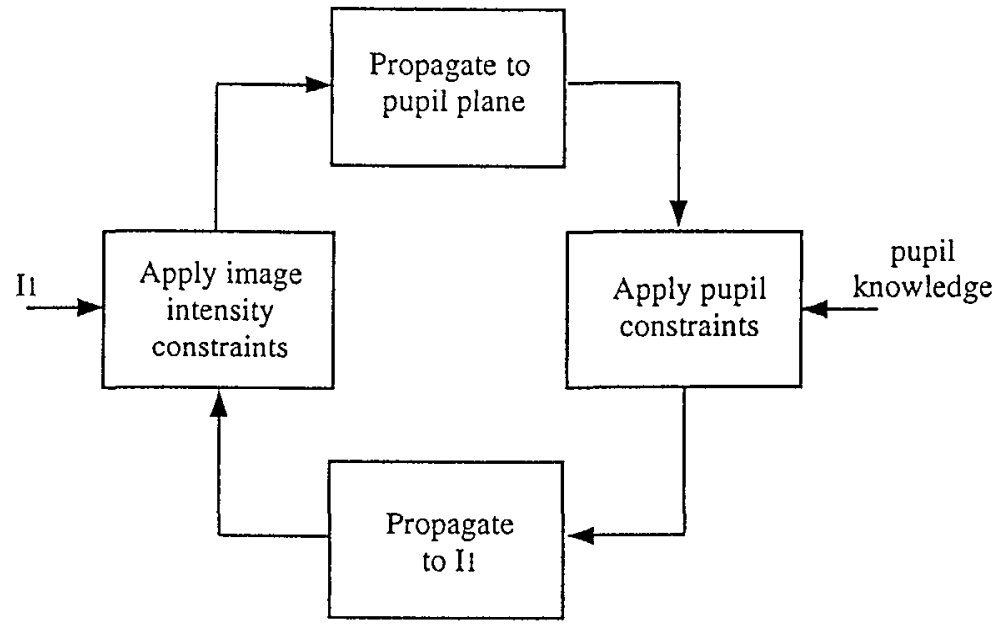

Figure 5. Block diagram of the error reduction method.

\subsubsection{Error reduction methods}

A block diagram describing the general approach to error reduction methods is depicted in Figure 5. A complex image of the pupil with random starting phase is propagated to the focal plane. Keeping the phase, the magnitude of the image is replaced by the magnitude of the measured intensity image. This new image is propagated back to the pupil plane, where the pupil constraints are applied to the magnitude. This process is continued for hundreds of iterations until the mean-squared-error between the measured and estimated intensity image reaches an acceptable level.

Despite the many iterations, this method runs very fast since the primary operations are single FFT's.

A variation of this method that we have found useful in our simulations and experiments is to measure $I_{1}$ at an out of focus position instead of at the focal plane. The same algorithm is used except that the propagation is performed to and from the out of focus position instead of the in focus position. Use of multiple images at several focal positions may further improve accuracy and convergence time. ${ }^{\ominus}$

\section{PHASE RETRIEVAL SIMULATIONS WITH ATMOSPHERIC TURBULENCE}

Even for the internal calibration of the AO system, there may be some atmospheric turbulence along the system optical path due to ambient air currents. This weak atmospheric turbulence can affect the accuracy of the phase retrieval algorithms. In other situations, it may be desirable to calibrate or assess the calibration of the AO system using an astronomical source that passes through the Earth's atmosphere. This could be done with the AO system either on or off. To determine the effect of atmospheric turbulence on the different phase retrieval techniques, we have performed simulations with atmospheric phase screens of varying total phase error along with a static phase error that we would like to detect and correct. 
Table 1. Parameters used in phase retrieval simulations.

\begin{tabular}{|l|l|}
\hline Aperture & Nyquist sampled, circular with $\mathrm{D}=3 \mathrm{~m}$ \\
\hline Static aberration & Combination of Zernikes $(\mathrm{Z} 3,4,5,7,11,15$, and 19$)$ \\
\hline Phase diversity & 6 PTV radians out of focus \\
\hline Screen size & $128 \times 128$ \\
\hline Object & Point source \\
\hline Wavelengths used & $0.7 \mu \mathrm{m}$ and $2.1 \mu \mathrm{m}$ \\
\hline Atmospheric coherence factors $\left(r_{0}\right.$ 's $)$ & $0.1 \mathrm{~m}$ and $10 \mathrm{~m}$ at $0.5 \mu \mathrm{m}$ \\
\hline Image exposure time & Long exposure $(6$ seconds with wind speed $=50 \mathrm{~m} / \mathrm{s})$ \\
\hline
\end{tabular}

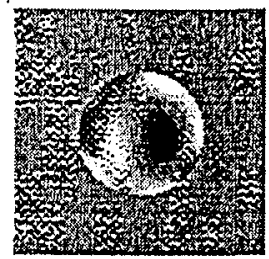

Figure 6. Static aberration applied to simulated images. The RMS phase variation is $97 \mathrm{~nm}$.

The simulation parameters we used are listed in Table 1. The adaptive optics was turned off for each case and then turned on for the $r_{0}=0.1 \mathrm{~m}$ cases. For the cases in which the AO was on, the DM used had an $8 \times 8$ actuator geometry, which corresponds to the Lick system.

The static aberration applied to the wavefront phase used to create the images is displayed in Figure 6.

The results of the weak turbulence simulations are shown in Figure 7 and the results of the strong turbulence simulations are shown in Figures 8 and 9.

The three methods described in the previous sections were applied to the simulated data: the least squares method(LS), the gradient search method(GS) assuming a Poisson noise model and point source object, and 200 iterations of the error reduction(ER) method using the out of focus image. Performance of the algorithm is measured as the RMS error in $\mathrm{nm}$ between the estimated and actual static wavefront aberration. The results are tabulated in Table 2.

Table 2. Performance comparison of phase retrieval algorithms.

\begin{tabular}{|ll|lll|c|}
\hline$r_{0}(\mathrm{~m})$ & & \multicolumn{3}{|c|}{ Performance } \\
at $0.5 \mu \mathrm{m}$ & $\lambda(\mu \mathrm{m})$ & LS & GS & ER & AO status \\
\hline 10 & 0.7 & 14 & 6 & 20 & OFF \\
10 & 2.1 & 42 & 13 & 52 & OFF \\
0.1 & 0.7 & $>97$ & $>97$ & $>97$ & OFF \\
0.1 & 2.1 & 71 & $>97$ & 87 & OFF \\
0.1 & 0.7 & 23 & $>97$ & $>97$ & ON \\
0.1 & 2.1 & 42 & $>97$ & 66 & ON \\
\hline
\end{tabular}

These results demonstrate that with weak turbulence, such as in a laboratory setting, it is possible to obtain static correction to below $20 \mathrm{~nm} \mathrm{rms}$ without adaptive optics running. The gradient search method appears to work the best for this case, which is probably largely due to the explicit point source assumption in that algorithm, while the least squares method is allowed to determine the object. With a weak atmosphere, the point source object still appears as a point source. The results also demonstrate that with a strong atmosphere, either longer wavelengths or adaptive optics or both are needed. The least squares method performed better with the strong atmosphere cases because it allows the object to become a blurred point source, which more closely matches the long exposure image 


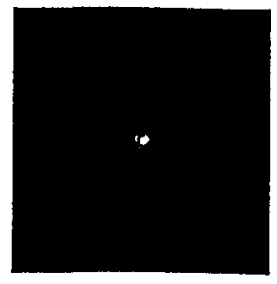

(a) In focus image, $\lambda=0.7 \mu \mathrm{m}$

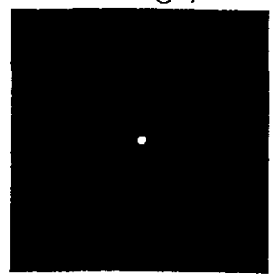

(c) In focus image, $\lambda=2.1 \mu \mathrm{m}$

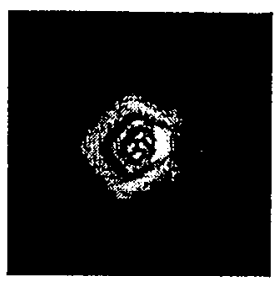

(b) Out of focus image, $\lambda=0.7 \mu \mathrm{m}$

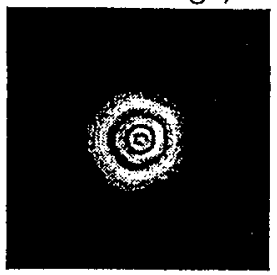

(d) Out of focus image, $\lambda=2.1 \mu \mathrm{m}$

Figure 7. Weak turbulence case $\left(r_{0}=10.0 \mathrm{~m}\right)$ images with AO turned off.

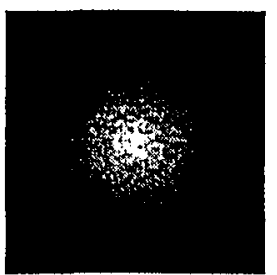

(a) In focus image, $\lambda=0.7 \mu \mathrm{m}$

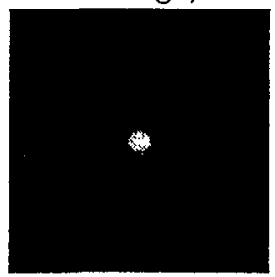

(c) In focus image, $\lambda=2.1 \mu \mathrm{m}$

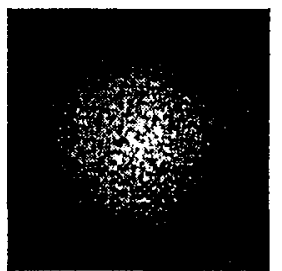

(b) Out of focus image, $\lambda=0.7 \mu \mathrm{m}$

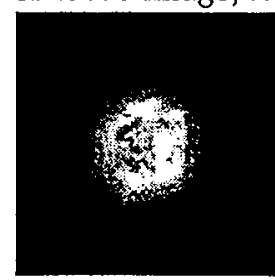

(d) Out of focus image, $\lambda=2.1 \mu \mathrm{m}$

Figure 8. Strong turbulence case $\left(r_{0}=0.1 \mathrm{~m}\right)$ images with $\mathrm{AO}$ turned off. 


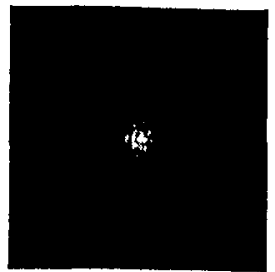

(a) In focus image, $\lambda=0.7 \mu \mathrm{m}$

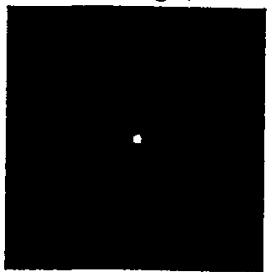

(c) In focus image, $\lambda=2.1 \mu \mathrm{m}$

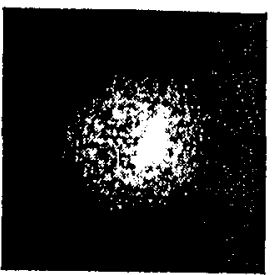

(b) Out of focus image, $\lambda=0.7 \mu \mathrm{m}$

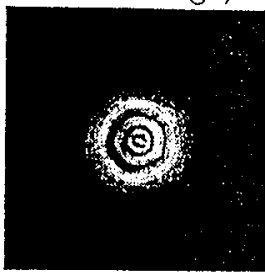

(d) Out of focus image, $\lambda=2.1 \mu \mathrm{m}$

Figure 9. Strong turbulence case $\left(r_{0}=0.1 \mathrm{~m}\right)$ images with $\mathrm{AO}$ turned on.

of a point source in strong turbulence. The gradient search routine would work better if the object was not assumed to be a point source.

\section{EXPERIMENTAL AO SYSTEM CALIBRATION USING PHASE RETRIEVAL}

Using the general procedure described in Section 2 to optimize the science camera image with an AO system, we have developed the following experimental steps:

- Measure science camera image(s).

- Estimate the wavefront phase using phase retrieval. We used the error reduction algorithm with the out of focus image.

- Impose basis set constraints. We used idealized Gaussian shaped DM influence functions.

- Translate estimated phase map into WFS offsets.

- Close the AO loop with the new offsets.

Using a point source calibration object, the science camera recorded in and out of focus images as shown in Figure 10. The out of focus image was then used in the error reduction phase retrieval algorithm to create the estimated wavefront phase map shown in Figure 11. It has an RMS phase error of 0.91 radians or $91 \mathrm{~nm}$.

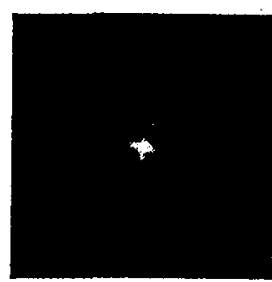

(a) In focus image, Strehl $=0.28$

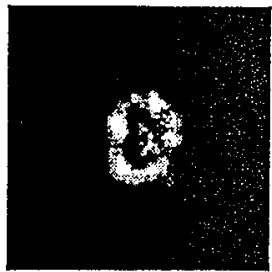

(b) Out of focus image

Figure 10. Original in and out of focus images obtained with science camera of point source calibration target. $(\lambda=0.633 \mu \mathrm{m})$

We fit the DM mirror modes to this phase map, resulting in 61 values which relate to a voltage for each actuator of the DM. This vector of 61 values is then multiplied by the system matrix resulting in 80 WFS pixel offsets ( $40 \mathrm{x}$, 


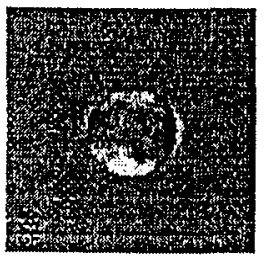

Figure 11. Estimated phase map using 200 iterations of error reduction algorithm. The RMS phase variation is 91 nm.

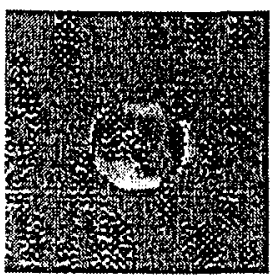

(a) Estimated phase map as fit by a basis set consisting of $61 \mathrm{DM}$ modes

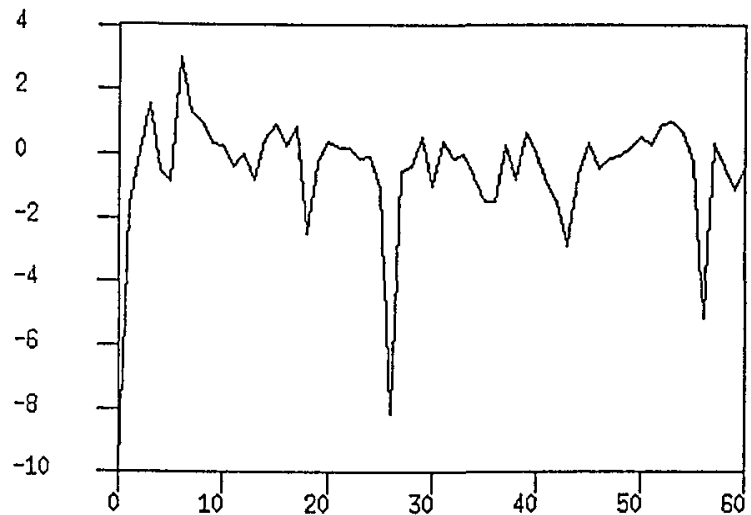

(b) Values of the $61 \mathrm{DM}$ coefficients.

The actuator number is on the $\mathrm{x}$-axis, and the $\mathrm{y}$-axis is in radians of phase with $\lambda=0.633 \mu \mathrm{m}$.

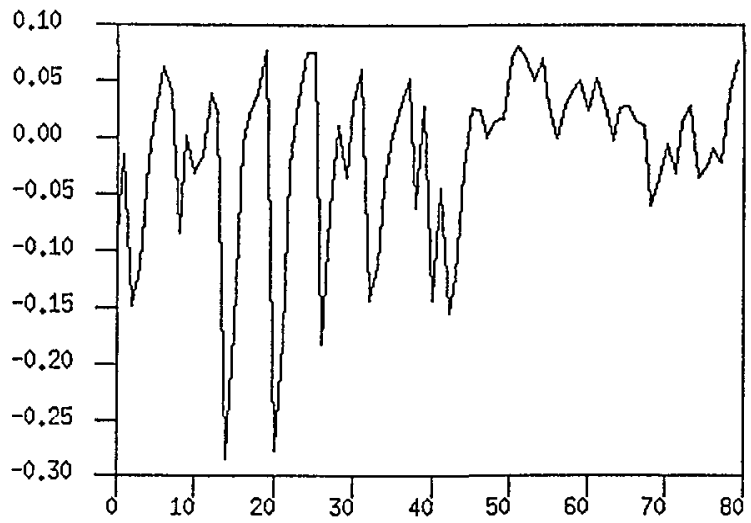

(c) Values of the WFS pixel offsets.

The subaperture number is on the $x$-axis and the $y$-axis is in pixels. ( 1 pixel $=8.73 \mu \mathrm{rad}$ )

Figure 12. 


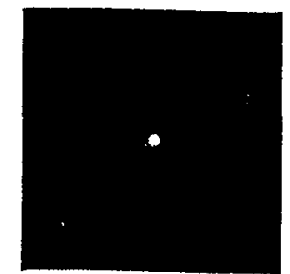

(a) In focus image, Strehl $=0.48$

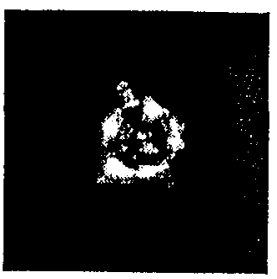

(b) Out of focus imagc

Figure 13. Improved in and out of focus images obtained with science camera of point source calibration target after calibration procedure. $(\lambda=0.633 \mu \mathrm{m})$

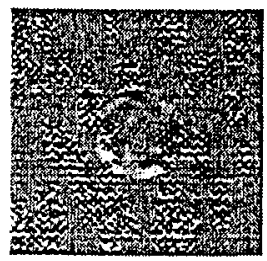

Figure 14. Estimated phase map using 200 iterations of error reduction algorithm. The RMS phase variation is 50 nm.

y pairs) which are fed directly into the closed-loop AO control system. The fitted phase map, vector of 61 values, and the vector of 80 WFS pixel offsets are displayed in Figure 12.

After closing the AO loop with the new WFS offsets, the science camera records the improved images displayed in Figure 13, indicating a Strehl ratio improvement of the in focus image from 0.28 to 0.48 . The estimated phase map shown in Figure 14 is now flatter with an rms phase error of 0.50 radians or $50 \mathrm{~nm}$. (Note: to be consistent with the Strehl value, there must be another 0.70 radians of rms phase error, duc to local turbulcnce or other crrors not accurately measured by the phase retrieval process.)

The current experimental results are not as good as the simulation results. We believe this can be attributed to several factors. A primary factor is the difference between the computed phase map and the phase applied by the DM. We are currently working on techniques to improve the translation from the computed phase map to the DM. Other factors contributing to the residual phase errors could be errors in the computed phase map from the phase retrieval or wavefront phase errors at spatial frequencies beyond the DM capability.

\section{CONCLUSIONS}

We have developed a phase retrieval based method for reducing the static aberrations in an AO system. Simulations with weak turbulence similar to that observed in the laboratory indicate that we should be able to reduce static aberrations to below the $20 \mathrm{~nm} \mathrm{rms}$ level of residual error. Simulations also predict that this method may be feasible using a star with the adaptive optics system running closed-loop on natural or laser guide stars. This could allow diagnosis and correction of quasi-static aberrations under actual astronomical observing conditions. Our experimental results show aberration reduction down to the $50 \mathrm{~nm}$ level. We expect to improve this by increasing the agreement between the computed and applied phase maps. Additional improvement may be obtained by decreasing ambient air turbulence in the system with better baffling.

\section{ACKNOWLEDGMENTS}

Work performed under the auspices of the U.S. Department of Energy by the Lawrence Livermore National Laboratory under contract No. W-7405-ENG-48.

\section{REFERENCES}

1. J. R. Fienup, J. C. Marron, T. J Schulz, and J. H. Seldin, "Hubble Space Telescope characterized by using phase-retrieval algorithms", Applied Optics, Vol. 32, No. 10, April 1993 
2. B. L. Ellerbroek, et. al., "Comparison of Shack-Hartmann wavefront sensing and phase-diverse phase retricval", Proceedings of SPIE, Vol 3126

3. C. E. Max, S. S. Olivier, H. W. Friedman, ct. al., "Image Improvement from a Sodium-Layer Laser Guide Star Adaptive Optics System", Science, Vol. 277, September 1997

4. R. W. Gerchberg and W. O. Saxton, Optik 35, 237-24 6 (1972)

5. R. A. Gonsalves, "Phase retrieval and diversity in adaptive optics", Opt. Eng. 21, 829-832 (1982)

6. J. R. Fienup, "Phase retrieval algorithms: a comparison", Applied Optics, Vol 21, No. 15, August 1982

7. R. G. Paxman, T. J. Schulz, and J. R. Fienup, "Joint estimation of object and aberrations by using phase diversity", J. Opt. Soc. Am. A/Vol. 9, No. 7, July 1992

8. M. G. Lofdahl and G. B. Scharmer, "Wavefront sensing and image $r$ estoration from focused and defocused solar images", Astron. Astrophys. Suppl . Ser. 107, 243-264 (1994)

9. D. C. Redding, et. al., "Wavefront sensing and control for a next generation space telescope", Proceedings of SPIE, Vol 3356 


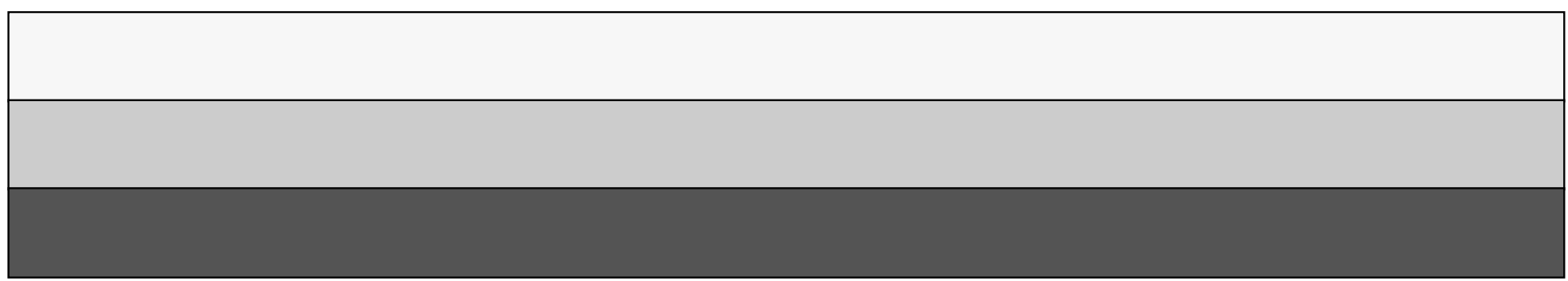

